Article

\title{
Numerical Solution of Nonlinear Fractional Diffusion Equation in Framework of the Yang-Abdel-Cattani Derivative Operator
}

\author{
Igor V. Malyk ${ }^{1}{ }^{\oplus}$, Mykola Gorbatenko ${ }^{2}{ }^{\oplus}$, Arun Chaudhary ${ }^{3}$, Shivani Sharma ${ }^{4}$ and Ravi Shanker Dubey ${ }^{4, *}$ \\ 1 Department of Mathematical Problems of Control and Cybernetics, Yuriy Fedkovych Chernivtsi National \\ University, Chernivtsi Region, 58012 Chernivtsi, Ukraine; i.malyk@chnu.edu.ua \\ 2 Department of Mathematical Modeling, Yuriy Fedkovych Chernivtsi National University, Chernivtsi Region, \\ 58012 Chernivtsi, Ukraine; m.gorbatenko@chnu.edu.ua \\ 3 Department of Mathematics, Rajdhani College, University of Delhi, Delhi 110015, India; \\ arunchaudhary@rajdhani.du.ac.in \\ 4 Department of Mathematics, AMITY School of Applied Science, AMITY University, Jaipur 302002, India; \\ shivani.sharma@s.amity.edu \\ * Correspondence: rsdubey@jpr.amity.edu
}

\section{check for} updates

Citation: Malyk, I.; Mykola, G.; Chaudhary, A.; Sharma, S.; Dubey, R.S. Numerical Solution of Nonlinear Fractional Diffusion Equation in the Framework of the Yang-Abdel-Cattan Derivative Operator. Fractal Fract. 2021, 5, 64. https://doi.org/10.3390/ fractalfract5030064

Academic Editors: Minghua Chen, Hossein Jafari, Can Li, Yajing Li and Lijing Zhao

Received: 24 May 2021

Accepted: 21 June 2021

Published: 2 July 2021

Publisher's Note: MDPI stays neutra with regard to jurisdictional claims in published maps and institutional affiliations.

Copyright: (c) 2021 by the authors. Licensee MDPI, Basel, Switzerland. This article is an open access article distributed under the terms and conditions of the Creative Commons Attribution (CC BY) license (https:// creativecommons.org/licenses/by/ $4.0 /)$.

\begin{abstract}
In this manuscript, the time-fractional diffusion equation in the framework of the Yang-AbdelCattani derivative operator is taken into account. A detailed proof for the existence, as well as the uniqueness of the solution of the time-fractional diffusion equation, in the sense of YAC derivative operator, is explained, and, using the method of $\alpha$-HATM, we find the analytical solution of the timefractional diffusion equation. Three cases are considered to exhibit the convergence and fidelity of the aforementioned $\alpha$-HATM. The analytical solutions obtained for the diffusion equation using the Yang-Abdel-Cattani derivative operator are compared with the analytical solutions obtained using the Riemann-Liouville (RL) derivative operator for the fractional order $\gamma=0.99$ (nearby 1 ) and with the exact solution at different values of $t$ to verify the efficiency of the YAC derivative operator.
\end{abstract}

Keywords: fractional derivative; existence and uniqueness; $\alpha$-homotopy analysis transform method MSC: Primary 92B05, 92C60; Secondary 26A33

\section{Introduction}

A parabolic partial differential equation that delineates the movement of energy and matter in a medium is called a diffusion equation. The diffusion process of heat or mass can be described using the concept of ordinary and partial derivatives. In today's era, fractional calculus [1-6] is emerging as an efficient and powerful tool in the field of science and technology. It is the branch of mathematics pertaining to the derivatives and integrals of arbitrary order and it is fruitful in explaining the concepts of damping, wave propagation and diffusion, biology, genetic algorithms, control systems, economy and finance, signal processing, robotics, system identification, electromagnetism, heat transfer, and many more. The literature is brimming with developments made in the field of fractional calculus. The most widely accepted definition including the singular kernel was proposed by Riemann and Liouville-Caputo. The next classification of fractional derivatives are made on the basis of the non-singular kernel. Caputo-Fabrizio proposed the first definition in the field of fractional calculus with a non-singular kernel [7]. Further development and success in the definitions of fractional derivatives were introduced by Prabhakar, Sonine, Wiman, Miller-Ross, Gorenglo, Mainardi, Mittag-Leffler, Atangana-Baleanu, Yang-Abdel-Cattani, and a lot more, who propose that the non-singular kernels are special functions, such as the Mittag-Leffler function, Miller-Ross function, Wiman function, Kohlrausch-William-Watts function, Rabotnov function, Prabhakar function, etc., see [8-13]. The fractional derivatives 
in which the non-singular kernels are the special functions are called general fractionalorder derivatives. Further applications of fractional derivatives can be seen in [14-27]. In this work, we will find the analytical solution of the following nonlinear fractional diffusion equation in the framework of the Yang-Abdel-Cattani (YAC) derivative operator.

$$
{ }_{0}^{Y A C} \mathcal{D}_{t}^{\gamma}(w(\eta, t))=\frac{\partial}{\partial \eta}\left(w^{a} \frac{\partial w(\eta, t)}{\partial \eta}\right) \quad w(\eta, 0)=w_{0},
$$

where ${ }_{0}^{Y A C} \mathcal{D}_{t}^{\gamma}(w(\eta, t))$ represents the Yang-Abdel-Cattani (YAC) fractional derivative of $w(\eta, t), w$ is the density of the diffusing medium at point $\eta$ and at time $t$. This generalized fractional derivative was presented by Yang et al. with the Rabotnov exponential function as the non-singular kernel. In this work, we will present a detailed proof for the existence as well as the uniqueness of the solution of the time-fractional diffusion equation in the framework of the YAC derivative operator and, using the $\alpha$-homotopy analysis transform method, we will find the analytical solution of the fractional diffusion equation in the sense of the YAC derivative operator. The analytical solutions obtained for the fractional diffusion equation using the YAC derivative operator are compared to the analytical solution obtained using the Riemann-Liouville (RL) derivative operator for the fractional order $\gamma=0.99$ (nearby 1 ) and with the exact solution at different values of $t$ to verify the efficiency of the YAC fractional derivative operator. Graphical representations of the analytical solutions are also given for a better understanding of the $\alpha$-HATM.

\section{Definitions}

Definition 1. [13] We define $\gamma, \chi \in \mathbb{R}^{+}$; the following series defines the Rabotnov exponential function of order $\gamma$

$$
\Psi\left(\chi u^{\gamma}\right)=\sum_{s=0}^{\infty} \frac{\chi^{s} u^{(s+1)(\gamma+1)-1}}{\Gamma((s+1)(\gamma+1))}, \quad u \in \mathbb{C}
$$

Definition 2. [13] For $k$ on $L^{1}[a, b], t>0, \chi \in \mathbb{R}^{+} 0<\gamma \leq 1$, the following defines the Yang-Abdel-Cattani fractional derivative of order $\gamma$

$$
{ }_{0}^{Y A C} \mathcal{D}_{t}^{\gamma}(k(t))=\int_{0}^{t} \Psi_{\gamma}\left(-\chi(t-\rho)^{\gamma}\right) k^{\prime}(\rho) d \rho .
$$

where, $\Psi_{\gamma}$ represents the Rabotnov exponential function of order $\gamma$.

Definition 3. [13] The following defines the Laplace transform for the Yang-Abdel-Cattani fractional derivative

$$
\mathcal{L}\left({ }_{0}^{\gamma A C} \mathcal{D}_{t}^{\gamma}(k(t))\right)=\frac{1}{p^{\gamma+1}} \frac{p \mathcal{L}[k(t)]-k(0)}{1+\chi p^{-(\gamma+1)}}
$$

Definition 4. [13] For $k \in L^{1}[a, b], t>0,0<\gamma \leq 1, \chi \in \mathbb{R}^{+}$, the following defines the fractional integral with Rabotnov fractional exponential function, of order $\gamma$

$$
I_{Y A C}^{\gamma} k(t)=\int_{0}^{t} \Psi_{\gamma}\left(-\chi(t-\rho)^{\gamma}\right) k(\rho) d \rho .
$$

Definition 5. [13] The following defines the Laplace transform for the Yang-Abdel-Cattani fractional integral

$$
\mathcal{L}\left(I_{Y A C}^{\gamma} k(t)\right)=\frac{1}{p^{\gamma+1}} \frac{\mathcal{L}(k(t))}{1+\chi p^{-(\gamma+1)}}
$$




\section{Existence of Solution of Fractional Diffusion Equation Using Yang-Abdel-Cattani Derivative Operator}

Theorem 1. Let us assume that the function $f\left(\eta, t, w, w^{\prime}, w^{\prime \prime}\right)$ satisfies the Lipschitz condition as

$$
\left|f\left(\eta, t, w, w^{\prime}, w^{\prime \prime}\right)-f\left(\eta, t, w_{1}, w_{1}^{\prime}, w_{1}^{\prime \prime}\right)\right| \leq M_{1}\left|w-w_{1}\right|+M_{2}\left|w^{\prime}-w_{1}^{\prime}\right|+M_{3}\left|w^{\prime \prime}-w_{1}^{\prime \prime}\right| .
$$

We also assume that

$$
\begin{aligned}
& \left|w^{\prime}-w_{1}^{\prime}\right| \leq k_{1}\left|w-w_{1}\right| \\
& \left|w^{\prime \prime}-w_{1}^{\prime \prime}\right| \leq k_{2}\left|w-w_{1}\right|
\end{aligned}
$$

where $k_{1}, k_{2} \in \mathbb{R}^{+}$then there exists a unique solution for the following time-fractional differential equation.

$$
{ }_{0}^{Y A C} \mathcal{D}_{t}^{\gamma}(w(\eta, t))=\frac{\partial}{\partial \eta}\left(w^{a} \frac{\partial w(\eta, t)}{\partial \eta}\right)
$$

Proof. We define

$$
\Phi(w, \eta)=f\left(\eta, t, w, w^{\prime}, w^{\prime \prime}\right)=\frac{\partial}{\partial \eta}\left(w^{a} \frac{\partial w(\eta, t)}{\partial \eta}\right)
$$

We first show that $\Phi(w, \eta)$ satisfies Lipschitz condition. Consider

$$
\begin{array}{r}
\left\|\Phi(w, \eta)-\Phi\left(w_{1}, \eta\right)\right\|=|| f\left(\eta, t, w, w^{\prime}, w^{\prime \prime}\right)-f\left(\eta, t, w_{1}, w_{1}^{\prime}, w_{1}^{\prime \prime}\right) \| \\
\leq M_{1}\left\|w-w_{1}\right\|+M_{2}\left\|w^{\prime}-w_{1}^{\prime}\right\|+M_{3}\left\|w^{\prime \prime}-w_{1}^{\prime \prime}\right\| \\
\leq M_{1}\left\|w-w_{1}\right\|+M_{2} k_{1}\left\|w-w_{1}\right\|+M_{3} k_{2}\left\|w-w_{1}\right\| \\
=\left(M_{1}+M_{2} k_{1}+M_{3} k_{2}\right)\left\|w-w_{1}\right\| .
\end{array}
$$

We also define $M_{1}+M_{2} k_{1}+M_{3} k_{2}=M$. So finally, we have

$$
\begin{array}{r}
\left\|\Phi(w, \eta)-\Phi\left(w_{1}, \eta\right)\right\|=\left\|f\left(\eta, t, w, w^{\prime}, w^{\prime \prime}\right)-f\left(\eta, t, w_{1}, w_{1}^{\prime}, w_{1}^{\prime \prime}\right)\right\| \\
\leq M\left|w-w_{1}\right|
\end{array}
$$

Using Picard's theorem, we obtain

$$
w(\eta, t)=w(\eta, 0)+\int_{0}^{t} \Psi_{\gamma}\left(-\chi(t-\rho)^{\gamma}\right) \Phi(w, \eta(\rho)) d \rho .
$$

For convenience, we write

$$
\int_{0}^{t} \Psi_{\gamma}\left(-\chi(t-\rho)^{\gamma}\right) \Phi(w, \eta(\rho)) d \rho=I_{Y A C}^{\gamma} \Phi(w, \eta)
$$

Finally, we have

$$
\begin{aligned}
w(\eta, t) & =w(\eta, 0)+I_{Y A C}^{\gamma} \Phi(w, \eta) \\
w(\eta, t)-w(\eta, 0) & =I_{Y A C}^{\gamma} \Phi(w, \eta) \\
\|w(\eta, t)-w(\eta, 0)\| & =\left\|I_{Y A C}^{\gamma} \Phi(w, \eta)\right\| \\
& =\left\|\int_{0}^{t} \Psi_{\gamma}\left(-\chi(t-\rho)^{\gamma}\right) \Phi(w, \eta(\rho)) d \rho .\right\| \\
& \leq \int_{0}^{t}\left\|\Psi_{\gamma}\left(-\chi(t-\rho)^{\gamma}\right)\right\|\|\Phi(w, \eta(\rho))\| d \rho . \\
& \leq\|\Phi(w, \eta(\rho))\| \int_{0}^{t} \Psi_{\gamma}\left(-\chi(t-\rho)^{\gamma}\right) \\
& =I_{Y A C}^{\gamma}(1)\|\Phi(w, \eta(\rho))\|
\end{aligned}
$$


As we proved that $\Phi(w, \eta)$ satisfies Lipschitz condition, so the following holds

$$
\|\Phi(w, \eta)\| \leq K
$$

Hence

$$
\|w(\eta, t)-w(\eta, 0)\| \leq K I_{Y A C}^{\gamma}(1)
$$

Finally, we consider

$$
\begin{aligned}
\left\|w(\eta, t)-w_{1}(\eta, t)\right\| & =\left\|I_{Y A C}^{\gamma} \Phi(w, \eta)-I_{Y A C}^{\gamma} \Phi\left(w_{1}, \eta\right)\right\| \\
& \leq I_{Y A C}^{\gamma}(1)\left\|\Phi(w, \eta)-\Phi\left(w_{1}, \eta\right)\right\| \\
& \leq I_{Y A C}^{\gamma} M\left\|w-w_{1}\right\|
\end{aligned}
$$

For the above map to be a contraction, we must have

$$
\begin{aligned}
M I_{Y A C}^{\gamma} & \leq 1 \\
I_{Y A C}^{\gamma} & \leq \frac{1}{M}
\end{aligned}
$$

Hence the existence and the uniqueness of the solution follows as a consequence of the Banach fixed point theorem.

\section{4. $\alpha$-HATM Solution of Nonlinear Time-Fractional Diffusion Equation}

Consider the nonlinear fractional diffusion equation, given as:

$$
{ }_{0}^{\gamma A C} \mathcal{D}_{t}^{\gamma}(w(\eta, t))=\frac{\partial}{\partial \eta}\left(w^{a} \frac{\partial w(\eta, t)}{\partial \eta}\right)
$$

We rewrite the above equation as

$$
{ }_{0}^{Y A C} \mathcal{D}_{t}^{\gamma}(w(\eta, t))-\left[a w^{a-1}\left(\frac{\partial w}{\partial \eta}\right)^{2}+w^{a} \frac{\partial^{2} w}{\partial \eta^{2}}\right]=0
$$

Taking the Laplace transform on the two sides of Equation (29),

$$
\frac{1}{p^{\gamma+1}}\left[\frac{p \mathcal{L}[w(\eta)]-w(\eta, 0)}{1+\chi p^{-(\gamma+1)}}\right]-\mathcal{L}\left[a w^{a-1}\left(\frac{\partial w}{\partial \eta}\right)^{2}+w^{a} \frac{\partial^{2} w}{\partial \eta^{2}}\right]=0
$$

By simplifying, we obtain

$$
\mathcal{L}[w(\eta, t)]-\frac{w(\eta, 0)}{p}-\left(\frac{p^{\gamma+1}+\chi}{p}\right) \mathcal{L}\left[a w^{a-1}\left(\frac{\partial w}{\partial \eta}\right)^{2}+w^{a} \frac{\partial^{2} w}{\partial \eta^{2}}\right]=0
$$

Let $\mathcal{N}$ be a nonlinear operator, defined as

$$
\begin{aligned}
& \mathcal{N}[\beta(\eta, t, \alpha)]= \\
& \mathcal{L}[\beta(\eta, t, \alpha)]-\frac{w(\eta, 0)}{p}-\left(\frac{p^{\gamma+1}+\chi}{p}\right) \mathcal{L}\left[a \beta^{a-1}\left(\frac{\partial \beta}{\partial \eta}\right)^{2}+\beta^{a} \frac{\partial^{2} \beta}{\partial \eta^{2}}\right]
\end{aligned}
$$

where $\beta(\eta, t, \alpha)$ is a function in $\eta, t, \alpha$, and $\alpha \in[0,1 / r]$ is an embedding parameter. Now construct the homotopy as

$$
(1-r \alpha) L\left[\beta(\eta, t, \alpha)-w_{0}(\eta, t)\right]=\alpha k \mathcal{N}[\beta(\eta, t, \alpha)]
$$

where $\beta(\eta, t, \alpha)$ is a function of $\eta, t$ and $\alpha, w_{0}(\eta, t)$ is an initial guess of $w(\eta, t), \mathcal{L}$ is the Laplace transform, and $k \neq 0$ is an auxiliary parameter. From above equation, we see that 
when $\alpha=0, \beta(\eta, t, 0)=w_{0}(\eta, t)$

when $\alpha=\frac{1}{r}, \beta(\eta, t, 1 / r)=w(\eta, t)$

This shows that as $\alpha$ varies from 0 to $1 / \mathrm{r}$, the solution $\beta(\eta, t, \alpha)$ changes from $w_{0}(\eta, t)$ the initial guess to $w(\eta, t)$ the exact solution.

Expand $\beta(\eta, t, \alpha)$ with respect to $\alpha$ using the Taylor series, we obtain

$$
\beta(\eta, t, \alpha)=w_{0}(\eta, t)+\sum_{i=1}^{\infty} w_{i}(\eta, t) \alpha^{i}
$$

where

$$
w_{i}(\eta, t)=\left.\frac{1}{i !} \frac{\partial^{i} \beta(\eta, t ; \alpha)}{\partial \alpha^{i}}\right|_{\alpha=0}
$$

Let $w_{0}(\eta, t), k, r$ be selected appropriately, the series defined in Equation (35) converges at $\alpha=1 / r$, hence

$$
w(\eta, t)=w_{0}(\eta, t)+\sum_{i=1}^{\infty} w_{i}(\eta, t)\left(\frac{1}{r}\right)^{i}
$$

Defining the vectors $\bar{w}_{n}=\left\{w_{0}, w_{1}, \ldots, w_{n}\right\}$, and differentiating Equation (33) i-times with respect to $\alpha$, and substituting $\alpha=0$, and lastly dividing them by $i$, we obtain:

$$
\mathcal{L}\left[w_{i}(\eta, t)-\zeta_{i} w_{i-1}(\eta, t)\right]=k \Re_{i}\left(w_{i-1}(\eta, t)\right),
$$

where

$$
\Re_{i}\left(w_{i-1}(\eta, t)\right)=\left.\frac{1}{(i-1) !} \frac{\partial^{i-1} N(\eta, t ; \alpha)}{\partial \alpha^{i-1}}\right|_{\alpha=0}
$$

and

$$
\zeta_{i}=\left\{\begin{array}{rr}
0, & i \leq 1 \\
r, & \text { otherwise }
\end{array}\right.
$$

Using Equations (32) and (38), we obtain

$$
\begin{aligned}
& \Re_{i}\left(w_{i-1}(\eta, t)\right)=L\left[w_{i-1}\right]-\frac{w(\eta, 0)}{p}\left(1-\frac{\zeta_{i}}{r}\right) \\
& \mathcal{L}\left[a w_{i-1}^{a-1}\left(\frac{\partial w_{i-1}}{\partial \eta}\right)^{2}+w_{i-1}^{a} \frac{\partial^{2} w_{i-1}}{\partial \eta^{2}}\right]
\end{aligned}
$$

Lastly, take the inverse Laplace transform on both sides of Equation (37),

$$
w_{m}(\eta, t)=\zeta_{i} w_{i-1}(\eta, t)+k \mathcal{L}^{-1}\left[\Re_{i}\left(w_{i-1}\right)\right] .
$$

Finally, opting for the suitable values of $k$ and $r$, the $\alpha$-HATM series solution is obtained, which is given as

$$
w(\eta, t)=\lim _{N \rightarrow \infty} \sum_{i=0}^{\infty} w_{i}(\eta, t)\left(\frac{1}{r}\right)^{i}
$$

We now consider different cases of the above nonlinear diffusion equation.

\subsection{Case 1}

We will now find the analytical solution of the following non-linear fractional diffusion equation in sense of YAC derivative operator using above mentioned $\alpha$ - HATM.

$$
{ }_{0}^{\gamma A C} \mathcal{D}_{t}^{\gamma}(v(\eta, t))=\frac{\partial}{\partial \eta}\left(\frac{\partial v(\eta, t)}{\partial \eta}\right), 0<t<1 ; 0<\gamma<1, \quad v(\eta, 0)=\sin (\pi \eta)
$$


Using the $\alpha$-HATM, the series solution is given as

$$
\begin{gathered}
v_{0}^{Y A C}(\eta, t)=\sin (\pi \eta) \\
v_{1}^{Y A C}(\eta, t)=k \pi^{2}\left(t \chi+\frac{t^{-\gamma}}{\Gamma(1-\gamma)}\right) \sin (\pi \eta) \\
v_{2}^{Y A C}(\eta, t)=r v_{1}^{Y A C}(\eta, t)+ \\
\frac{1}{2} k^{2} \pi^{2}\left[\frac{2 \pi^{2} t^{-2 \gamma}}{\Gamma(1-2 \gamma)}+\frac{2 t^{-\gamma}}{\Gamma(1-\gamma)} t \chi\left(2+\pi^{2} t \chi+\frac{4 \pi^{2} t^{-\chi}}{\Gamma(2-\gamma)}\right)\right] \sin (\pi \eta)
\end{gathered}
$$

The following diffusion equation's

$$
\frac{\partial v(\eta, t)}{\partial t}=\frac{\partial^{2} v(\eta, t)}{\partial \eta^{2}}
$$

exact solution is given as

$$
\sin (\pi \eta) e^{-\pi^{2} t}
$$

In Table 1, we will compare the $\alpha$-HATM solutions obtained for Case 1 in terms of the YAC fractional derivative operator with the analytical solutions obtained using the Riemann-Liouville fractional derivative operator [28] and with the considered diffusion equation's exact solution at different values of $t$ for $\eta=0.25$ and $\gamma=0.99$ ( $\gamma$ close to 1$)$ $k=-0.01, r=3$ and $\chi=3$.

Table 1. Comaprison of $\alpha$ - HATM Solution in sense of YAC derivative operator, RL derivative operator and exact solution for Case 1.

\begin{tabular}{cccccc}
\hline$t$ & $v(R L)$ & $v(Y A C)$ & $v($ Exact $)$ & Error $($ RL $)$ & Error $($ YAC $)$ \\
\hline 0.1 & 0.196571 & 0.263464 & 0.263544 & $6.6973 \times 10^{-2}$ & $8 \times 10^{-5}$ \\
\hline 0.2 & 0.902091 & 0.0981688 & 0.098225 & $8.0159 \times 10^{-3}$ & $5.62 \times 10^{-5}$ \\
\hline 0.3 & 0.0303086 & 0.0361351 & 0.0366092 & $6.3006 \times 10^{-3}$ & $4.741 \times 10^{-4}$ \\
\hline 0.4 & 0.0165324 & 0.0131774 & 0.0136445 & $2.8879 \times 10^{-3}$ & $4.671 \times 10^{-4}$ \\
\hline 0.5 & 0.00463999 & 0.00507924 & 0.00508543 & $4.4544 \times 10^{-4}$ & $6.19 \times 10^{-6}$ \\
\hline 0.6 & 0.00134119 & 0.00188248 & 0.00189538 & $5.5419 \times 10^{-4}$ & $1.29 \times 10^{-5}$ \\
\hline 0.7 & 0.000591754 & 0.000645395 & 0.000706423 & $1.14669 \times 10^{-4}$ & $6.1028 \times 10^{-5}$ \\
\hline 0.8 & 0.000208632 & 0.000240379 & 0.000263289 & $5.4657 \times 10^{-5}$ & $2.291 \times 10^{-5}$ \\
\hline 0.9 & 0.0000935588 & 0.0000971667 & 0.00009813 & $4.5712 \times 10^{-6}$ & $9.633 \times 10^{-7}$ \\
\hline 1.0 & 0.0000300947 & 0.0000351593 & 0.0000365738 & $6.4791 \times 10^{-6}$ & $1.4145 \times 10^{-6}$ \\
\hline
\end{tabular}

In Figure 1, the 3-D plots of the $\alpha$-HATM solution for the YAC operator are compared to the exact solution for $\gamma=0.99(\gamma$ nearby 1$)$.

\subsection{Case 2}

We will now find the analytical solution of the following non-linear fractional diffusion equation in sense of YAC derivative operator using above mentioned $\alpha-$ HATM.

$$
{ }_{0}^{Y A C} \mathcal{D}_{t}^{\gamma}(v(\eta, t))=\frac{\partial}{\partial \eta}\left(\frac{v^{2} \partial v(\eta, t)}{\partial \eta}\right), 0<\gamma<1 ; 0<t<1, \quad v(\eta, 0)=\frac{\eta+b}{c}
$$


Using the $\alpha$-HATM, the series solution is given as

$$
\begin{gathered}
v_{0}^{Y A C}(\eta, t)=\frac{\eta+b}{c} \\
v_{1}^{Y A C}(\eta, t)=\frac{-k(b+\eta)}{4 c^{3}}\left(t \chi+\frac{t^{-\gamma}}{\Gamma(1-\gamma)}\right)
\end{gathered}
$$

$$
\begin{aligned}
& v_{2}^{Y A C}(\eta, t)=r v_{1}^{Y A C}(\eta, t)+\frac{k^{2} t^{-2 \gamma}(b+\eta)}{4 c^{5} \Gamma(1-2 \gamma) \Gamma(1-\gamma) \Gamma(2-\gamma)} \\
& \quad\left[2 \Gamma(1-\gamma) \Gamma(2-\gamma)+t^{\gamma} \Gamma(1-2 \gamma)\left(-c^{2} \Gamma(1-2 \gamma)+t \chi \Gamma(1-\gamma)\left[4-t^{\gamma}\left(c^{2}-t \chi\right) \Gamma(2-\gamma)\right]\right)\right]
\end{aligned}
$$

The following diffusion equation's

$$
\frac{\partial v(\eta, t)}{\partial t}=\frac{\partial}{\partial \eta}\left(\frac{v^{2} \partial v(\eta, t)}{\partial \eta}\right)
$$

exact solution is given as

$$
\frac{\eta+b}{2 \sqrt{c^{2}-t}}, t \leq c^{2}
$$

In Table 2, we will compare the $\alpha$-HATM solutions obtained for Case 2 in terms of the YAC fractional derivative operator with the analytical solutions obtained in terms of the Riemann-Liouville fractional derivative operator [28] and with the considered diffusion equation's exact solution at different values of $\mathrm{t}$ for $k=-0.19, r=1, \chi=1, b=2, c=3$, $\eta=0.25$ and $\gamma=0.99$.

Table 2. Comaprison of $\alpha$ - HATM Solution in sense of YAC derivative operator, RL derivative operator and exact solution for Case 2.

\begin{tabular}{cccccc}
\hline$t$ & $v(R L)$ & $v($ YAC $)$ & $v($ Exact $)$ & Error $(R L)$ & Error $($ YAC $)$ \\
\hline 0.1 & 0.362916 & 0.377039 & 0.377101 & $1.4185 \times 10^{-2}$ & $6.2 \times 10^{-5}$ \\
\hline 0.2 & 0.375416 & 0.377759 & 0.379237 & $3.821 \times 10^{-3}$ & $1.478 \times 10^{-3}$ \\
\hline 0.3 & 0.379474 & 0.381023 & 0.381411 & $1.937 \times 10^{-3}$ & $3.88 \times 10^{-4}$ \\
\hline 0.4 & 0.380159 & 0.383075 & 0.383622 & $3.463 \times 10^{-3}$ & $5.47 \times 10^{-4}$ \\
\hline 0.5 & 0.382443 & 0.384292 & 0.3858872 & $3.4442 \times 10^{-3}$ & $1.58 \times 10^{-3}$ \\
\hline 0.6 & 0.387566 & 0.388041 & 0.388162 & $5.96 \times 10^{-4}$ & $1.21 \times 10^{-4}$ \\
\hline 0.7 & 0.388769 & 0.390407 & 0.390493 & $1.724 \times 10^{-3}$ & $8.6 \times 10^{-5}$ \\
\hline 0.8 & 0.39034 & 0.392672 & 0.392867 & $2.527 \times 10^{-3}$ & $1.72 \times 10^{-4}$ \\
\hline 0.9 & 0.390757 & 0.395034 & 0.395285 & $4.528 \times 10^{-3}$ & $2.51 \times 10^{-4}$ \\
\hline 1.0 & 0.405465 & 0.397722 & 0.397748 & $7.717 \times 10^{-3}$ & $2.6 \times 10^{-5}$ \\
\hline
\end{tabular}

In Figure 2, the 3-D plots of the $\alpha$-HATM solution for the YAC operator are compared to the exact solution for $\gamma=0.99(\gamma$ nearby 1$)$.

\subsection{Case 3}

We will now find the analytical solution of the following non-linear fractional diffusion equation in sense of YAC derivative operator using above mentioned $\alpha-$ HATM.

$$
{ }_{0}^{\gamma A C} \mathcal{D}_{t}^{\gamma}(v(\eta, t))=\frac{\partial}{\partial \eta}\left(\frac{v^{-2} \partial v(\eta, t)}{\partial \eta}\right), 0<t<1 ; 0<\gamma<1, \quad v(\eta, 0)=\frac{1}{\sqrt{1+\eta^{2}}}
$$


Using the $\alpha$-HATM, the series solution is given as

$$
\begin{gathered}
v_{0}^{Y A C}(\eta, t)=\frac{1}{\sqrt{1+\eta^{2}}} \\
v_{1}^{Y A C}(\eta, t)=-k\left[\frac{\eta^{2}}{\left(1+\eta^{2}\right)^{3 / 2}}-\frac{1}{\sqrt{1+\eta^{2}}}\right]
\end{gathered}
$$

The following diffusion equation's

$$
\frac{\partial v(\eta, t)}{\partial t}=\frac{\partial}{\partial \eta}\left(\frac{v^{-2} \partial v(\eta, t)}{\partial \eta}\right)
$$

exact solution is

$$
\frac{1}{\sqrt{\eta^{2}+e^{2 t}}}
$$

In Table 3, we will compare the $\alpha$-HATM solutions obtained for Case 3 in terms of the YAC fractional derivative operator with the analytical solutions obtained in terms of the Riemann-Liouville fractional derivative operator [28] and with the considered diffusion equation's exact solution at different values of $\mathrm{t}$ for $k=-0.6, r=1, \chi=1, \eta=0.25$, and $\gamma=0.99$.

Table 3. Comaprison of $\alpha$ - HATM Solution in sense of YAC derivative operator, RL derivative operator and exact solution for Case 3.

\begin{tabular}{cccccc}
\hline$t$ & $v(R L)$ & $v(Y A C)$ & $v($ Exact $)$ & Error $(R L)$ & Error $($ YAC $)$ \\
\hline 0.1 & 0.842589 & 0.861515 & 0.882539 & $3.995 \times 10^{-2}$ & $2.1024 \times 10^{-2}$ \\
\hline 0.2 & 0.780412 & 0.805378 & 0.802101 & $2.1689 \times 10^{-2}$ & $3.277 \times 10^{-3}$ \\
\hline 0.3 & 0.698454 & 0.723338 & 0.728431 & $2.9972 \times 10^{-2}$ & $5.093 \times 10^{-3}$ \\
\hline 0.4 & 0.640023 & 0.64123 & 0.661101 & $2.1078 \times 10^{-2}$ & $1.9871 \times 10^{-2}$ \\
\hline 0.5 & 0.589874 & 0.559094 & 0.599676 & $9.802 \times 10^{-3}$ & $4.058 \times 10^{-3}$ \\
\hline 0.6 & 0.521176 & 0.553665 & 0.543718 & $2.254 \times 10^{-2}$ & $9.947 \times 10^{-3}$ \\
\hline 0.7 & 0.462397 & 0.484287 & 0.492802 & $3.0405 \times 10^{-2}$ & $8.515 \times 10^{-3}$ \\
\hline 0.8 & 0.460085 & 0.45875 & 0.446521 & $1.3564 \times 10^{-2}$ & $1.23 \times 10^{-2}$ \\
\hline 0.9 & 0.428753 & 0.394834 & 0.404486 & $2.4267 \times 10^{-2}$ & $9.652 \times 10^{-3}$ \\
\hline 1.0 & 0.387562 & 0.330926 & 0.366333 & $2.1229 \times 10^{-2}$ & $3.24 \times 10^{-2}$ \\
\hline
\end{tabular}

In Figure 3, the 3-D plots of the $\alpha$-HATM solution for the YAC operator are compared to the exact solution for $\gamma=0.99(\gamma$ nearby 1$)$.

\section{Conclusions}

In this paper, the time-fractional nonlinear diffusion equation is taken into consideration in regards to the Yang-Abdel-Cattani fractional derivative operator. The $\alpha$-HATM is used to find the analytical solution of the nonlinear fractional diffusion equation. The analytical solutions obtained from the $\alpha$-HATM, in the sense of the YAC derivative operator, are compared to the analytical solutions obtained via the Riemann-Liouville derivative operator and with the exact solution for all the three cases at distinct values of time $t$, and we observe that the analytical solutions obtained using the YAC derivative operator coincide with the exact solution more closely as compared to the Riemann-Liouville derivative operator when the value of fractional order is close to 1 , i.e., $\gamma=0.99$. In Figures $1-3$ we plotted the 3-D representations of the solution $v(\eta, t)$ with the exact solution for case 1 , 
case 2, and case 3, respectively. In Figures 1-3a, the 3-D representation of the analytical solution attained using $\alpha$-HATM along with the exact solution is given. In Figures $1-3 b$, the $\alpha$-HATM solutions are plotted for different values of $k$ along with the exact solution and we see that the $\alpha$-HATM solution is in best fit with the exact solution for a suitable value of $k$ and hence $k=-0.01$ works as an optimal value for case $1, k=-0.19$ works as an optimal value for case 2 , and $k=-0.6$ works as an optimal value for case 3 . In Figures $1-3 c$, the $\alpha$-HATM solutions are plotted for different values of $r$ along with the exact solution and we see that the $\alpha$-HATM solution is in best fit with the exact solution for a suitable value of $r$ and hence $r=3$ works as an optimal value for case 1 and $r=1$ works as an optimal value for cases 2 and 3 . In Figures 1-3d, the $\alpha$-HATM solutions are plotted for different values of $\chi$ along with the exact solution and we see that the $\alpha$-HATM solution is in best fit with the exact solution for a suitable value of $\chi$ and hence $\chi=3$ works as an optimal value for case 1 and $\chi=1$ works as optimal value for case 2 and 3 . Hence, we see the efficient role of the parameters $k, r$ provided in the $\alpha$-HATM, and $\chi$, given in the YAC definition in regulating the convergence of the solution. Hence we see that YAC fractional derivative operator provides better results than the Riemann-Liouville fractional derivative operator.

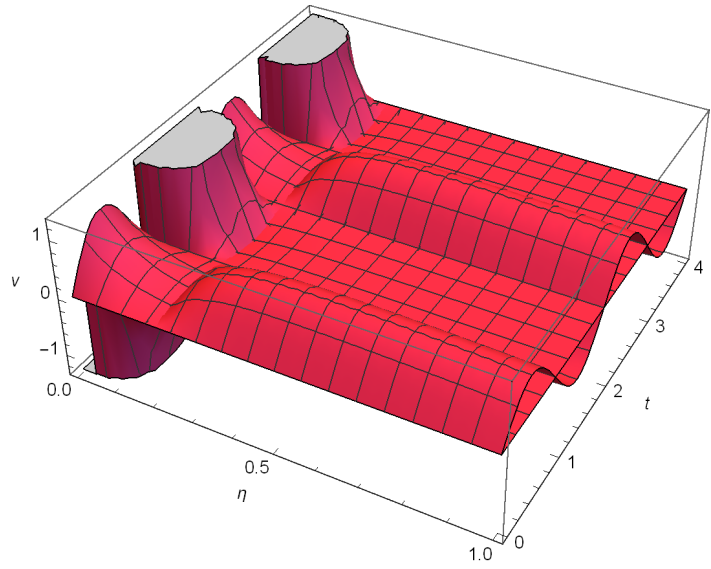

(a)

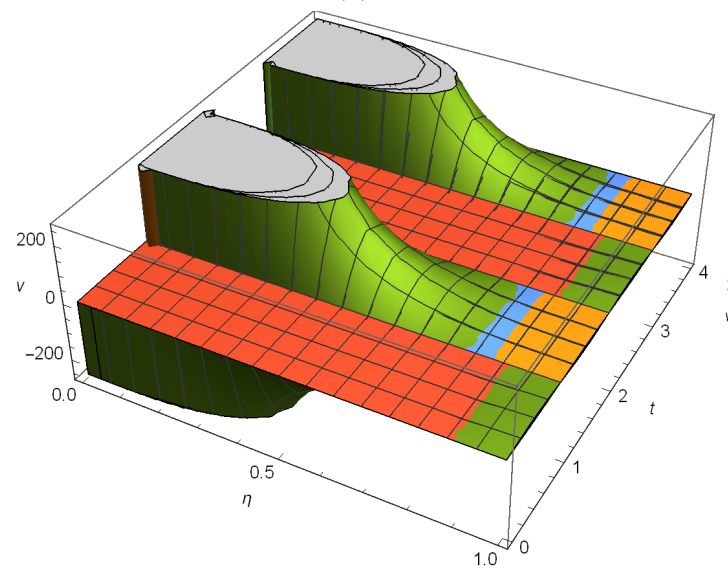

(c)

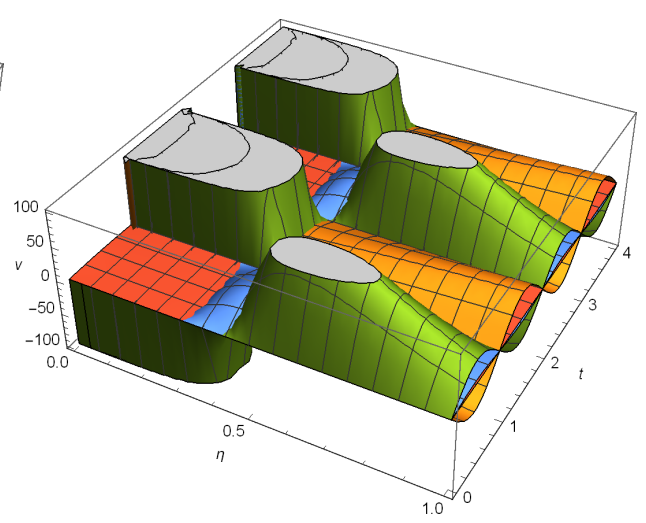

(b)

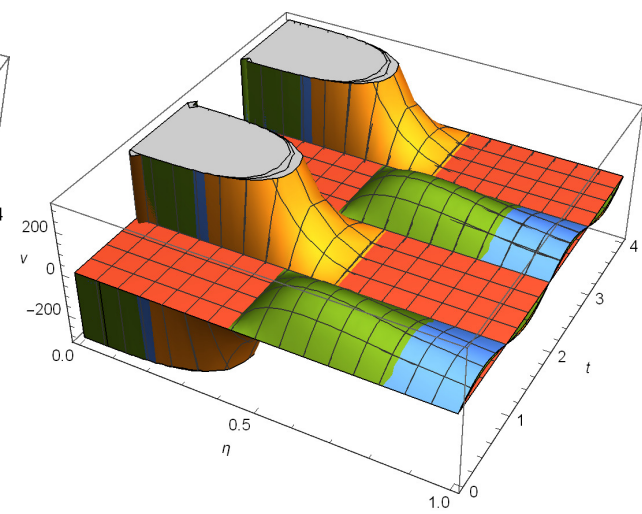

(d)

Figure 1. 3-D Plot of $\alpha$-HATM solution for YAC operator with exact solution for case 1 when $\gamma=0.99$. (a) $\alpha$-HATM solution with exact solution. (b) $\alpha$-HATM solution with exact solution for distinct values of $k$, Yellow $(\mathrm{k}=-0.01)$, Blue $(\mathrm{k}=-0.04)$, Green $(\mathrm{k}=-0.08)$, Red (Exact Solution). (c) $\alpha$-HATM solution with exact solution for distinct values of $r$, Yellow $(\mathrm{r}=1)$, Blue $(\mathrm{r}=2)$, Green $(\mathrm{r}=3)$, Red (Exact Solution). (d) $\alpha$-HATM solution with exact solution for distinct values of $\chi$, Yellow $(\chi=3)$, Blue $(\chi=5)$, Green $(\chi=7)$, $\operatorname{Red}($ Exact Solution). 


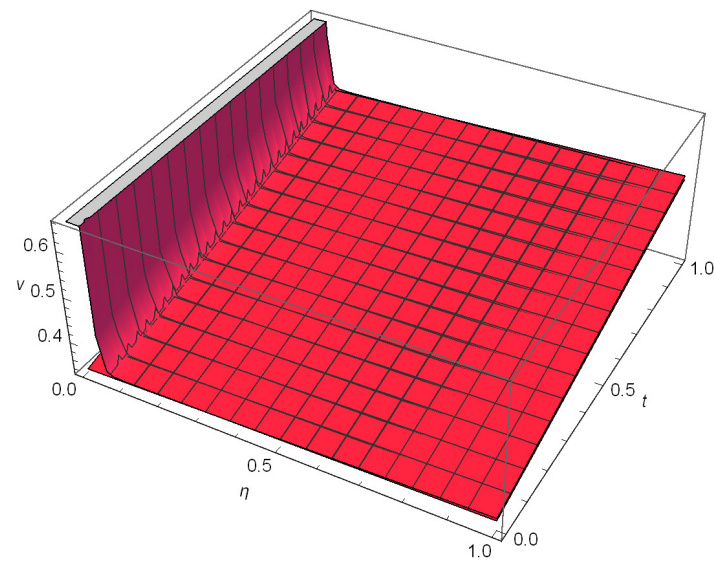

(a)

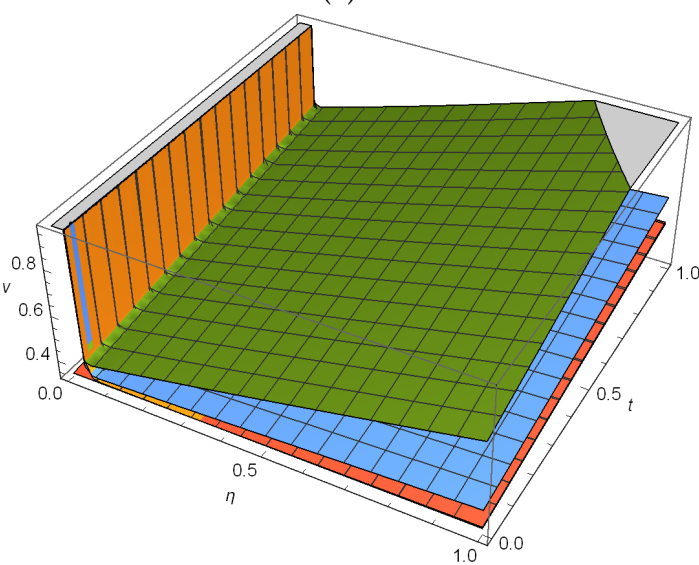

(c)

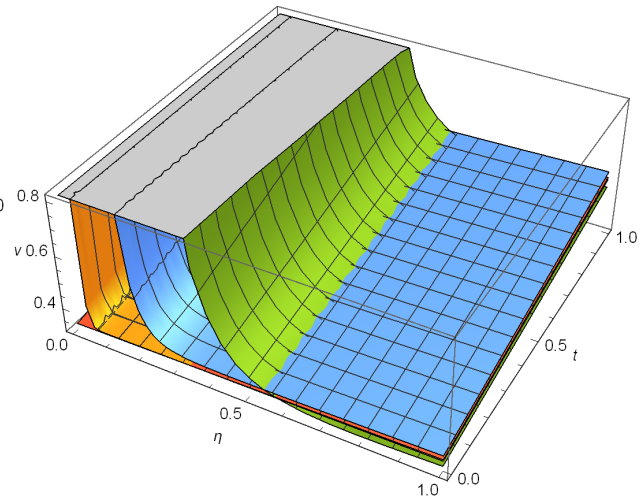

(b)

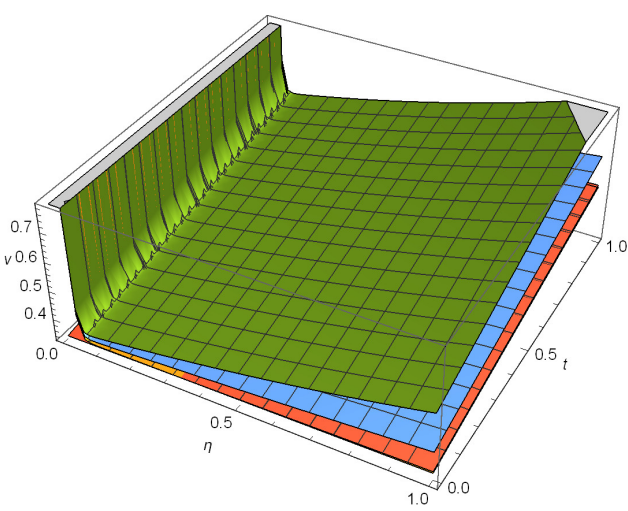

(d)

Figure 2. 3-D Plot of $\alpha$-HATM solution for YAC operator with exact solution for case 2 when $\gamma=0.99$. (a) $\alpha$-HATM solution with exact solution. (b) $\alpha$-HATM solution with the exact solution for distinct values of $\mathrm{k}$, Yellow $(\mathrm{k}=-0.19)$, Blue $(\mathrm{k}=-1)$, Green $(\mathrm{k}=-2)$, Red (Exact Solution). (c) $\alpha$-HATM solution with exact solution for distinct values of $r$, Yellow $(r=1)$, Blue $(r=2)$, Green $(r=3)$, Red (Exact Solution). (d) $\alpha$-HATM solution with exact solution for distinct values of $\chi$, Yellow $(\chi=1)$, Blue $(\chi=5)$, Green $(\chi=10)$, Red (Exact Solution).

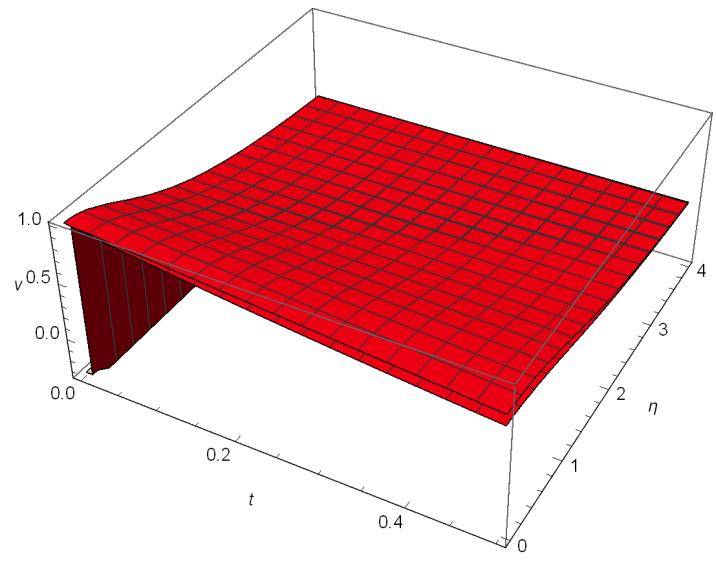

(a)

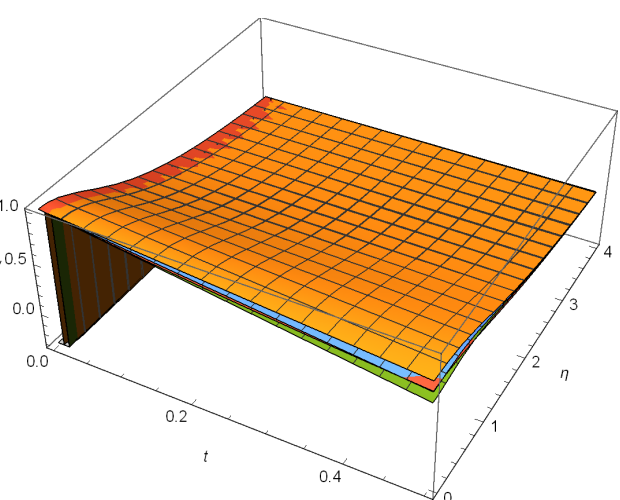

(b)

Figure 3. Cont. 


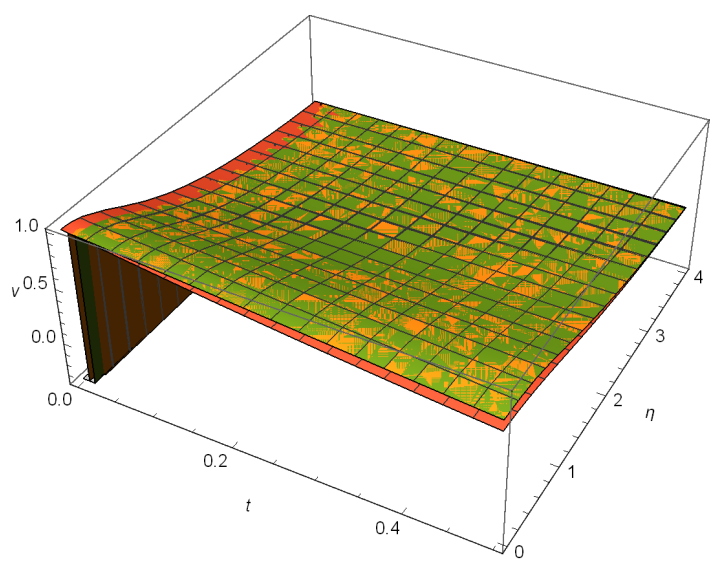

(c)

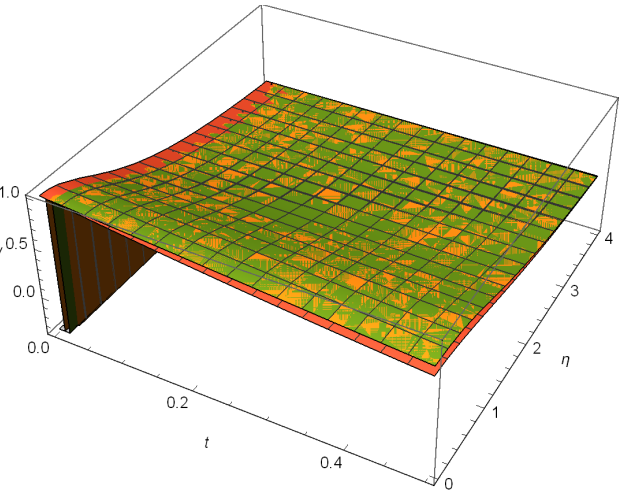

(d)

Figure 3. 3-D Plot of $\alpha$-HATM solution for YAC operator with exact solution for case 3 when $\gamma=0.99$. (a) $\alpha$-HATM solution with exact solution. (b) $\alpha$-HATM solution with exact solution for distinct values of $\mathrm{k}$, Yellow $(\mathrm{k}=-0.6)$, Blue $(\mathrm{k}=-0.8)$, Green $(\mathrm{k}=-1)$, Red (Exact Solution). (c) $\alpha$-HATM solution with exact solution for distinct values of $r$, Yellow $(r=1)$, Blue $(r=2)$, Green $(r=3)$, Red (Exact Solution). (d) $\alpha$-HATM solution with exact solution for distinct values of $\chi$, Yellow $(\chi=1)$, Blue $(\chi=2)$, Green $(\chi=3)$, Red (Exact Solution).

Author Contributions: Conceptualization, I.V.M., M.G., S.S. and R.S.D.; methodology, S.S. and R.S.D.; software, S.S. and R.S.D.; validation, I.V.M., M.G., A.C. and R.S.D.; formal analysis, I.V.M., M.G., A.C. and R.S.D.; writing-original draft preparation, S.S. and R.S.D.; writing-review and editing, S.S., R.S.D. and A.C.; supervision, I.V.M., M.G., A.C. and R.S.D.; funding acquisition, I.V.M. All authors have read and agreed to the published version of the manuscript.

Funding: The APC was funded by Igor V. Malyk.

Institutional Review Board Statement: Not applicable.

Informed Consent Statement: Not applicable.

Data Availability Statement: Not applicable.

Acknowledgments: We would like to express our sincere thanks to the reviewers for their valuable comments and suggestions.

Conflicts of Interest: The authors declare no conflict of interest.

\section{References}

1. Miller, K.S.; Ross, B. An Introduction to the Fractional Calculus and Fractional Differential Equations; A Wiley-Interscience Publication; John Wiley and Sons, Inc.: New York, NY, USA, 1993; p. xvi+366, ISBN 0-471-58884-9.

2. Oldham, K.B.; Spanier, J. The Fractional Calculus Theory and Applications of Differentiation and Integration to Arbitrary Order; With an Annotated Chronological Bibliography by Bertram Ross; Academic Press: New York, NY, USA; London, UK, 1974; Volume 111, p. xiii+234, ISBN 9780125255509.

3. Kilbas, A.A.; Srivastava, H.M.; Trujillo, J.J. Theory and Applications of Fractional Differential Equations; North-Holland Mathematics Studies; Elsevier Science B.V.: Amsterdam, The Netherlands, 2006; Volume 204, p. xvi+523, ISBN 978-0-444-51832-3.

4. Podlubny, I. Fractional Differential Equations: An Introduction to Fractional Derivatives, Fractional Differential Equations, to Methods of Their Solution and Some of Their Applications; Academic Press, Inc.: San Diego, CA, USA,1999; Volume 198, p. xxiv+340, ISBN 0-12-558840-2.

5. Zhou, Y. Basic Theory of Fractional Differential Equations; World Scientific Publishing Co. Pte. Ltd.: Hackensack, NJ, USA, 2014; p. $\mathrm{x}+293$, ISBN 978-981-4579-89-6. [CrossRef]

6. Yang, X.J. Advanced Local Fractional Calculus and Its Applications; World Science: New York, NY, USA, $2012 ;$ ISBN 139781938576010.

7. Caputo, M.; Fabrizio, M. A New Definition of Fractional Derivative without Singular Kernel. Prog. Fract. Differ. Appl. 2015, 1, 73-85. [CrossRef]

8. Atangana, A.; Baleanu, D. New fractional derivatives with nonlocal and nonsingular kernel: Theory and application to heat transfer model. Therm. Sci. 2016, 20, 763-769. [CrossRef]

9. Khalil, R.; Horani, M.A.; Yousef, A.; Sababheh, M. A new definition of fractional derivative. J. Comput. Appl. Math. 2014, 264, 65-70. [CrossRef] 
10. Jarad, F.; Abdeljawad, T. Generalized fractional derivatives and Laplace transform. Discret. Contin. Dyn. Syst.-S 2020, 13, 709-722. [CrossRef]

11. Yang, X.J. General Fractional Derivatives: Theory, Methods and Applications; Chapman and Hall/ CRC: Boca Raton, FL, USA, 2019; ISBN 9781138336162.

12. Yang, X.J.; Gao, F.; Ju, Y. General Fractional Derivatives with Applications in Viscoelasticity; Academic Press: Cambridge, MA, USA 2020; ISBN 9780128172087.

13. Yang, X.J.; Abdel-Aty, M.; Cattani, C. A new general fractional order derivative with Rabotnov fractional-exponential kernel applied to model the anomalous heat. Therm. Sci. 2019, 23, 1677-1681. [CrossRef]

14. Atangana, A. On the new fractional derivative and application to non-linear Fisher's reaction-diffusion equation. Appl. Math. Comput. 2016, 273, 948-956. [CrossRef]

15. Aguilar, J.P.; Korbel, J.; Luchko, Y. Application of the Fractional Diffusion Equation to Option Pricing and Risk Calculations. Mathematics 2019, 7, 796. [CrossRef]

16. Sontakke, B.R.; Shelke, A.S. Appropriate Scheme for Time fractional Diffusion Equation and Its Applications. Glob. J. Pure Appl. Math. 2019, 13, 4333-4345.

17. Constantinescu, C.D.; Ramirez, J.M.; Zhu, W.B. An application of fractional differential equations to risk theory. Math. Stochastics 2019, 23, 1001-1024. [CrossRef]

18. Ali, K.K.; Osman, M.S.; Baskonus, H.C.; Elazabb, N.S.; İlhan, E. Analytical and numerical study of the HIV-1 infection of CD4 ${ }^{+}$ T-cells conformable fractional mathematical model that causes acquired immunodeficiency syndrome with the effect of antiviral drug therapy. Math. Methods Appl. Sci. 2020. [CrossRef]

19. Gao, W.; Ghanbari, B.; Baskonus, H.C. New numerical simulations for some real world problems with Atangana-Baleanu fractional derivative. Chaos Solitons Fractals 2019, 128, 34-43. [CrossRef]

20. Ghanbari, B.; Gómez-Aguilar, J.F. Modelling the dynamics of nutrient-phytoplankton-zooplankton system with variable-order fractional derivatives. Chaos Solitons Fractals 2018, 116, 114-120. [CrossRef]

21. Kumar, S.; Ghosh, S.; Samet, B.; Goufo, E.F.D. An analysis for heat equations arises in diffusion process using new Yang-AbdelAty-Cattani fractional operator. Math. Methods Appl. Sci. 2020, 1-19. [CrossRef]

22. Jleli, M.; Kumar, S.; Kumar, R.; Samet, B. Analytical approach for time fractional wave equations in the sense of Yang-Abdel-AtyCattani via the homotopy perturbation transform method. Alex. Eng. J. 2019. [CrossRef]

23. Zhang, X.; Chen, Y.Q. Admissibility and robust stabilization of continuous linear singular fractional order systems with the fractional order $\alpha$ : The $0<\alpha<1$ case. ISA Trans. 2018, 82, 42-50. [CrossRef] [PubMed]

24. Belmor, S.; Ravichandran, C.; Jarad, F. Nonlinear generalized fractional differential equations with generalized fractional integral conditions. J. Taibah Univ. Sci. 2020. [CrossRef]

25. Tarasov, V.E. Fractional nonlinear dynamics of learning with memory. Non-Linear Dyn. 2020, 100, 1231-1242. [CrossRef]

26. Shone, T.T.; Patra, A. Solution for non-linear fractional partial differential equations using fractional complex transform. Int. J. Appl. Comput. Math. 2019, 5, 90. [CrossRef]

27. Tarasov, V.E. Exact solutions of Bernoulli and Logistic fractional differential equations with power law coefficients. Mathematics 2020, 8, 2231. [CrossRef]

28. Dubey, R.S.; Goswami, P. Analytical solution of the nonlinear diffusion equation. Eur. Phys. J. Plus 2018, 133. [CrossRef] 